Tropical Journal of Pharmaceutical Research December 2018; 17 (12): 2489-2495

ISSN: 1596-5996 (print); 1596-9827 (electronic) (1) Pharmacotherapy Group, Faculty of Pharmacy, University of Benin, Benin City, 300001 Nigeria.

\title{
Effect of Qianliexin capsule on quality of life, NIH-CPSI score and IIEF-5 score in patients with prostatitis complicated with erectile dysfunction
}

\author{
Chen Xiaosong ${ }^{1}$, Wang Ping ${ }^{2}$ \\ ${ }^{1}$ Urinary Department of Xuanwu Hospital, Capital Medical University, ${ }^{2}$ General Practice in Health Service Center, Baizhifang \\ Community of Xicheng District, Beijing City 100054, China
}

*For correspondence: Email: zv1169@163.com

Sent for review: 17 September 2018

Revised accepted: 27 November 2018

\begin{abstract}
Purpose: To study the influence of Qianliexin capsule on quality of life, NIH-CPS Index, and 5-item version of the 15-item International Index of Erectile Function (IIEF-5) scores in patients with prostatitis complicated with erectile dysfunction (ED).

Methods: Patients with prostatitis complicated with ED (128) who were treated from November 2015 to December 2017 participated in this study. The patients were assigned to two groups (64 per group) according to the treatment given: study group which was given routine treatment plus Qianliexin capsule, and control group which received routine treatment only. Clinical effectiveness, adverse reactions, Quality of Life-Bref (QOL-BREF) scores, etc, of the two groups were determined.

Results: The QOL-BREF score of the study group was significantly higher than that of the control group $(p<0.05)$. While $\mathrm{NIH}$-CPSI scores were significantly decreased in both groups, the decrease in the study group was lower $(p<0.05)$. In contrast, IIEF-5 score significantly increased in both groups, but the increase in the study group was higher than in control $(p<0.05)$.

Conclusion: Qianliexin capsule is an effective and reasonably safely drug for enhancing the quality of life, NIH-CPSI and IIEF-5 scores, as well as the lecithin bodies in prostatitis patients with ED.
\end{abstract}

Keywords: Qianliexin capsule, Prostatitis, Erectile dysfunction NIH-CPSI score, IIEF-5 score

\begin{abstract}
This is an Open Access article that uses a funding model which does not charge readers or their institutions for access and distributed under the terms of the Creative Commons Attribution License (http://creativecommons.org/licenses/by/4.0) and the Budapest Open Access Initiative (http://www.budapestopenaccessinitiative.org/read), which permit unrestricted use, distribution, and reproduction in any medium, provided the original work is properly credited.

Tropical Journal of Pharmaceutical Research is indexed by Science Citation Index (SciSearch), Scopus, International Pharmaceutical Abstract, Chemical Abstracts, Embase, Index Copernicus, EBSCO, African Index Medicus, JournalSeek, Journal Citation Reports/Science Edition, Directory of Open Access Journals (DOAJ), African Journal Online, Bioline International, Open-J-Gate and Pharmacy Abstracts
\end{abstract}

\section{INTRODUCTION}

Prostatitis is a common disease in the urinary surgery department. The main clinical symptoms are chronic pelvic pain and irritation in the urethra. The incidence of prostatitis has been on the increase in recent years. According to reports $[1,2]$, the incidence of prostatitis of patients over 25 years old is up to $35 \%$. Pathological studies have demonstrated that in prostatitis, cell infiltration occurs after local inflammation, and it involves glandular tube and surrounding interstitial tissue [3-5]. Due to closeness of the prostate to vessels involved in penile erection, prostatitis is associated with congestion, edema, and dilated glandular duct. If the inflammation is not promptly controlled, it will spread to surrounding tissues and even the whole gland, producing prostatic hyperplasia, fibrosis and calcification, all of which adversely influence 
penile erection. Thus, prostatitis patients often experience varying degrees of erectile dysfunction. In addition, the abnormal spermiation and mental stress caused by erectile dysfunction also aggravate symptoms of prostatitis, making recovery difficult $[6,7]$. It is believed that Qianliexin capsule, as a bloodregulating recipe, can activate blood to resolve stasis, removing heat and dampness, all of which are beneficial to the recovery of prostatitis patients with ED [8]. However, there are no studies so far on the influence of this capsule on QOL-BREF, NIH-CPSI and IIEF-5. The present study investigated the effect of Qianliexin capsule treatment on QOL-BREF, NIH-CPSI and IIEF-5 of prostatitis patients with ED.

\section{EXPERIMENTAL}

\section{Patient profile}

A total of 128 patients with prostatitis complicated with ED treated from November 2015 to December 2017 were selected as study subjects. There were two groups (64 patients per group) based on the method of treatment used: study and control groups. The study group patients were aged from 20 to 60 years (mean age $=34.2$ years), with disease course ranging from 5 to 62 months (mean disease course = 15.3 months). Patients in the control group were within the age range of 21 - 59 years (mean age $=33.7$ years), with disease course of 5 to 59 months. Approval for the study was received from the Ethical Committee of Urinary Department of Xuanwu Hospital, Capital Medical University (approval no. 20188723), and complied with the guidelines of Declaration of Helsinki promulgated in 1964 as amended in 1996 [9].

\section{Inclusion criteria}

The following patients were included: patients who met the diagnostic criteria of prostatitis and ED; patients whose ages were from 23 to 52 years; and patients who had normal sex life over 6 months before the treatment. Other included patients were those with normal liver function; patients who signed informed consent with their family members, and patients whose conditions were not accompanied with stones in ureters and urinary bladder.

\section{Exclusion criteria}

Patients who had severe mental diseases, and those whose ages were less than 23 years or over 52 years, as well as patients with malformation or stenosis in urinary tract were excluded. Other excluded patients included those with incomplete hospital data, and patients who had benign prostatic hypertrophy. Age and disease course in the two groups were comparable $(p>0.05)$.

\section{Diagnostic criteria}

\section{Prostatitis}

The clinical symptoms were lower urinary tract symptoms such as odynuria, frequent micturition, urgent urination and scorching heat in urinary tract; inflammatory or pain symptoms such as pain in perineum, bearing-down and distending lumbosacral pain, and white secretion in urinary tract. The second set of symptoms were based on palpation texture of prostate: tenderness, inflammatory nodes, full gland bod and even textures in hardness. The third set of symptoms involved results from EPS microscopic examination: decreased lecithin bodies or their complete disappearance, and WB $\geq 10 / \mathrm{HP}$. Patients who met any of the features listed in these three diagnostic criteria were diagnosed with prostatitis.

\section{Erectile dysfunction (ED)}

IIEF-5 score less than 22 points was diagnosed as ED. Furthermore, IIEF-5 with 12 - 21 points was classified as mild, IIEF-5 with 8 to 11 points was categorized as moderate, while IIEF- 5 with 5 to 7 points was taken as severe.

\section{Criteria for treatment effectiveness}

The therapeutic effects were classified into three grades: recovery, effective and ineffective. If the symptoms of prostatitis completely disappeared and IIEF-5 score was over 22 points, recovery was implied; effective referred to $25-50 \%$ decreases in IIEF-5 score, while NIH-CPSI less than $25 \%$ and IEF-5 score below $25 \%$ meant ineffective.

The total effectiveness $=[($ recovery score + effective score)/ total score] $\times 100 \%$.

\section{Treatment plan}

Patients in two groups were given advice and guidance on living habits, as well as routine treatment with antibody and $\alpha 1$ receptor antagonist. The control group was given levofloxacin (Shangyu Xinjing Pharmaceutical Co., Ltd, SFDA, approval number H20103650) at a dose of $0.2 \mathrm{~g}$ twice daily for $4-6$ consecutive weeks, and $2 \mathrm{mg}$ hytrin (Shanghai Yanan Pharmaceutical Co., Ltd, SFDA approval number 
H20103431) once every night for 2 consecutive months. Both treatments were given via the oral route. The study group was given $2 \mathrm{~g}$ of Qainliexin capsule (Jinan Hongjitang Co., Ltd, SFDA approval number Z10950010) thrice daily for 2 consecutive months.

\section{Enzyme-linked immunosorbent assay}

After suppressing sensual passion, the external orifice of the urethra was disinfected, and $1 \mathrm{ml}$ of prostatic fluid was collected after massage and then preserved at $-80{ }^{\circ} \mathrm{C}$ prior to use. Doubleantibody sandwich avidinbiotincomplex-enzymes linked immunosorbent assay (ABC-ELISA) was used for the assay of IFN- $\gamma$ and IL-8 levels in prostatic fluid. One drop of prostatic fluid was smeared on a slide and subjected to routine examination for WBC number and lecithin body number.

\section{Questionnaire assessment}

After 2 months of treatment, questionnaire methods and QOL-BREF were used to assess psychological health and life quality of patients in both groups. The assessments covered four aspects of social relationships, psychology, environment and physiology.

\section{Therapeutic indices}

The indices used were therapeutic effects and adverse reactions, NIH-CPSI scores, IIEF-5 scores, and QOL-BREF scores, WBC number, lecithin body number, IFN-y and IL-8 levels, and correlation between NIH-CPSI scores and IIEF-5 scores of ED patients in both groups. All of these indices are the common ones used to evaluate the therapeutic effects.

\section{Statistical analysis}

Independent sample $t$-test was used for comparing $\mathrm{NIH}-\mathrm{CPSI}, \mathrm{IIEF}-5$ and QOL-BREF scores, as well as WBC, lecithin body number, and IFN-y and IL-8 levels in prostatic fluid. Chi square test was used to compare therapeutic effects and adverse reactions between the two groups, while correlation analysis was done using Person relevance. All analyses were done with SPSS version 22.0. Values of $p<0.05$ were considered statistically significant.

\section{RESULTS}

\section{Therapeutic outcomes}

As shown in Table 1, there were 2 recovery cases (3.13\%), 11 effective cases (17.19\%), and 51 ineffective cases (79.69\%) in the control group, accounting for 13 total effective cases (20.31\%). In the study group, there were 14 recovery cases (21.88\%), 34 effective cases (53.13\%), and 16 ineffective cases (25\%), resulting in 48 total effective cases (75\%). The total effectiveness in the study group was superior to that of the control group $(p<0.05)$.

\section{IIEf-5 and NIH-CPSI scores of patients}

Before treatment, the NIH-CPSI and IIEF-5 scores in both groups were comparable. However, after 2 months of treatment, NIH-CPSI scores were lower in the two groups than corresponding scores before treatment, but $\mathrm{NIH}$ CPSI score in the study group was significantly less, relative to control value $(p<0.05$; Table 2$)$. Moreover, study group IIEF-5 score increased significantly post-treatment $(p<0.05)$.

Table 1: Treatment outcomes (n, \%)

\begin{tabular}{lccccc}
\hline Group & Cases & Recovery & Effective & Ineffective & Total effectiveness (\%) \\
\hline Control & 64 & $2(3.13)$ & $11(17.19)$ & $51(79.69)$ & $13(20.31)$ \\
Study & 64 & $14(21.88)$ & $34(53.13)$ & $16(25.00)$ & $48(75.00)$ \\
$\chi^{2}$ & & & & 38.366 \\
\hline$p$ & & & $<0.001$ \\
\hline
\end{tabular}

Table 2: NIH-CPSI and IIEF-5 scores of patients (mean \pm SD)

\begin{tabular}{|c|c|c|c|c|}
\hline \multirow[t]{2}{*}{ Group } & \multicolumn{2}{|c|}{ NIH-CPSI } & \multicolumn{2}{|c|}{ IIEF-5 } \\
\hline & Pre- treatment & Post-treatment & Pre- treatment & Post-treatment \\
\hline Control $(n=64)$ & $25.09 \pm 5.49$ & $18.47 \pm 5.88^{*}$ & $11.29 \pm 4.49$ & $12.78 \pm 5.19$ \\
\hline Study $(n=64)$ & $25.48 \pm 5.27$ & $11.38 \pm 5.68^{* *}$ & $10.78 \pm 3.77$ & $19.49 \pm 6.51^{* *}$ \\
\hline
\end{tabular}

${ }^{*} p<0.05$, vs pre-treatment, ${ }^{\star *} p<0.05$, vs control 


\section{QOL-BREF scores of patients}

Table 3 shows that after 2 months of treatment, physiological, psychological, social relationship, and environmental scores were $55.39 \pm 11.68$, $56.69 \pm 11.28,57.08 \pm 12.77$ and $55.23 \pm 13.88$ points, respectively for control group. For study group, physiological, psychological, social relationship, and environmental scores were $67.48 \pm 14.87,63.39 \pm 14.21,69.78 \pm 13.28$, and $66.38 \pm 14.18$ points, respectively. The QOLBREF scores in the study group were superior to those in the control group $(p<0.05)$.

\section{WBC count and lecithin body number}

Before treatment, WBC count and number of lecithin bodies of patients in both groups were comparable $(p>0.05)$. However, after 2 months of treatment, WBC counts were significantly in both groups, but was lower in the study group than in controls $(p<0.05)$. Lecithin body numbers in two groups increased significantly increased post-treatment, but was more in the study group than in control group ( $p<0.05)$, as shown in Table 4.

Table 3: QOL-BREF scores of patients (mean \pm SD)

\section{IFN-y and IL-8 levels of patients}

Table 5 shows that pre-treatment, the levels of IFN-y and IL-8 in both groups were similar $(p>$ 0.05 ). Two months after treatment, IFN-Y and IL8 levels in two groups significantly decreased, but they were lower in the study group than in controls $(p<0.05)$.

\section{Adverse reactions}

In the control group, there were 7 cases of vertigo $(10.94 \%), 8$ cases of dry stool $(12.5 \%)$, 6 cases of diarrhea $(9.38 \%)$, and 9 cases of vomiting (14.06\%), resulting in a total of 30 adverse reactions (46.88 \%) in the control group (Table 6). There were 3 cases of vertigo (4.69 $\%), 2$ cases of dry stool $(3.13 \%), 1$ case of diarrhea (1.56\%), and 4 cases of vomiting (6.25 $\%$ ), accounting for a total of 10 adverse reactions (15.63\%). The adverse reactions in the study group $(15.63 \%)$ was much lower than that in the control group $(46.88 \%, p<0.05)$.

\begin{tabular}{lcccc}
\hline Group & Physiology & Psychology & Social relationships & Environment \\
\hline Control $(n=64)$ & $55.39 \pm 11.68$ & $56.69 \pm 11.28$ & $57.08 \pm 12.77$ & $55.23 \pm 13.88$ \\
Study $(n=64)$ & $67.48 \pm 14.87^{*}$ & $63.39 \pm 14.21^{*}$ & $69.78 \pm 13.28^{* *}$ & $66.38 \pm 14.18(1)$ \\
\hline
\end{tabular}

${ }^{\star} P<0.05$, compared with control group

Table 4: WBC count and lecithin body number of patients (mean \pm SD)

\begin{tabular}{lcccc}
\hline Group & \multicolumn{2}{c}{ WBC (count/HP) } & \multicolumn{2}{c}{ Lecithin body (number/HP) } \\
\cline { 2 - 5 } & Pre-treatment & Post-treatment & Pre-treatment & Post-treatment \\
\hline Control $(\mathrm{n}=64)$ & $44.36 \pm 8.23$ & $17.52 \pm 6.55 \cup 1$ & $2.03 \pm 1.02$ & $2.53 \pm 1.05^{\star}$ \\
Study $(\mathrm{n}=64)$ & $45.12 \pm 8.02$ & $10.83 \pm 1.45^{\star *}$ & $2.08 \pm 0.98$ & $3.05 \pm 0.96^{\star *}$ \\
\hline${ }^{\star} P<0.05$, vs control & & &
\end{tabular}

${ }^{\overline{ }} P<0.05$, vs control

Table 5: IFN-Y and IL-8 levels of patients (mean \pm SD)

\begin{tabular}{lcccc}
\hline Group & \multicolumn{2}{c}{ IFN-y(ng/L) } & \multicolumn{2}{c}{ IL-8(ng/L) } \\
\cline { 2 - 5 } & Pre-treatment & Post-treatment & Pre-treatment & Post-treatment \\
\hline Control $(\mathrm{n}=64)$ & $20.66 \pm 7.52$ & $13.86 \pm 5.23^{*}$ & $228.36 \pm 35.67$ & $178.11 \pm 36.71^{*}$ \\
Study $(\mathrm{n}=64)$ & $21.86 \pm 6.89$ & $7.52 \pm 1.39^{* *}$ & $225.62 \pm 38.52$ & $156.15 \pm 43.56^{\star *}$ \\
\hline${ }^{*} P<0.05$, compared with the control group $^{* \star}{ }^{*} \mathrm{p}<0.05$ & &
\end{tabular}

${ }^{\star} P<0.05$, compared with the control group, ${ }^{\star \star} p<0.05$

Table 6: Adverse reactions of patients ( $n, \%)$

\begin{tabular}{lcccccc}
\hline Group & $\begin{array}{c}\text { No. of } \\
\text { cases }\end{array}$ & Vertigo & Dry stool & Diarrhea & Vomiting & $\begin{array}{c}\text { Total adverse } \\
\text { reactions }\end{array}$ \\
\hline Control & 64 & $7(10.94)$ & $8(12.5)$ & $6(9.38)$ & $9(14.06)$ & $30(46.88)$ \\
Study & 64 & $3(4.69)$ & $2(3.13)$ & $1(1.56)$ & $4(6.25)$ & $10(15.63)$ \\
$X^{2}$ & & & & & 14.546 \\
$p$ & & & & & $<0.001$ \\
\hline
\end{tabular}


Analysis of relationship between IIEF-5 and NIH-CPSI scores

The results of overall correlation analysis of the 128 ED cases revealed that NIH-CPSI scores were negatively correlated with IIEF-5 scores $(r=$ $-0.612, p<0.001$; Figure 1).

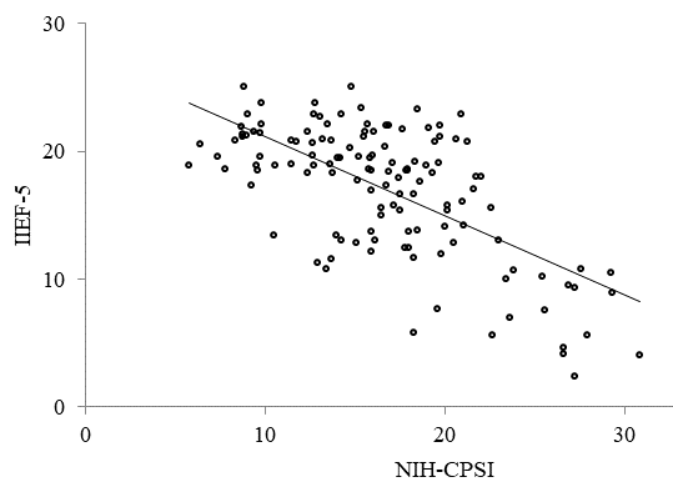

Figure 1: Correlation between NIH-CPSI and IIEF-5 scores

\section{DISCUSSION}

The etiology of prostatitis is multi-factorial and the mechanism is complicated. Pathogen infection, oxidative stress, abnormal immunoreaction, psychological factor, and lower urinary tract epithelial dysfunction are predisposing factors to prostatitis $[10,11]$. However, it is believed that the main pathogenic factor is pathogen infection due to Pseudomonas and Escherichia coli [12]. Pathogens invade the prostate gland through the urine, thereby inducing infection. This can lead to complicated and variable symptoms such as urinary infection, ED and infertility. Antibacterial drugs are the drugs of first choice in the treatment of prostatitis with ED caused by pathogen infection [5].

Studies have reported that Qainliexin capsule, a Chinese herbal preparation, can promote the levels of testicular hormones and boost secretion of the prostate gland by softening fibrosis tissue, and nourishing yin and replenishing kidney $[13,14]$. The capsule can relieve swelling and pain, clear heat, promote diuresis, and prevent and treat prostate hypertrophy, thereby significantly ameliorating prostatitis with ED. [15] It has been shown that Qianliexin capsule produces better curative effect in prostatitis patients than the same dose carbostyril, and it also produces less adverse reactions. Thus, investigating the effect of Qianliexin capsule on prostatitis patients with ED can provide a new direction for the treatment of this disease while enhancing the prognosis of patients.
Qianliexin capsule is composed of Flos carthami (Honghua), Radix paeoniae rubra (Chishao), Commiphora (Moyao), radix Salviae miltiorrhiza (Danshen) and Hirsute shiny Bugleweed (Zelan). This herbal combination can activate the blood and resolve stasis, promote testosterone hormone level, enhance secretion of the prostate gland, and clear heat and relieve strangury. Qianliexin capsule can relieve inflammation, and prevent and treat prostate hypertrophy caused by testosterone propionate. Multiple clinical studies have shown that treatment of ED patients with Qianliexin capsule results in good compliance and significant effects. The capsule has been shown to exert anti-inflammatory effects in chronic prostatitis patients through its influence on levels of inflammatory cytokines in WBC and neutrophils [16]. Clinical trials have revealed that Qianliexin capsule is well tolerated, and can restore prostate function in IIIA prostatitis patients and improve sperm activity [17].

The results obtained in this investigation showed that NIH-CPSI, IIEF-5 and QOL-BREF scores in the study group were significantly enhanced by treatment with Qianliexin capsule. In addition, the capsule produced low adverse reactions, which is basically consistent with previous studies $[16,17]$. It also improved the life quality of patients and the effects were better than those produced in the routine treatment group. Integrative correlation analysis showed that $\mathrm{NIH}$ CPSI scores were negatively associated with IIEF-5. The NIH-CPSI scores of patients decreased with increase in IIEF-5 scores, which shows a negative correlation between $\mathrm{NIH}-\mathrm{CPSI}$ scores and IIEF-5.

There are many research efforts based on the application of Qainliexin capsule as therapy for prostatitis with ED, but only few have focused on its influence on WBC count, lecithin body number, and IFN- $\gamma$ and IL-8 levels in prostatic fluid. The WBCs are also called immune cells, and they consist of eosinophils, basophilic granulocytes and neutrophils. They prevent diseases by functioning as phagocytes. They surround and engulf invading pathogenic bacteria in the seminal vesicle, seminiferous duct, epididymis and testis. Therefore, an increase in WBC number denotes increase in inflammation. The WBC count of a healthy adult is $4.0-10.0 \times 10^{9} / \mathrm{L}$, and it increases rapidly when chronic or acute tissue infections occur. The WBC count is positively correlated with the degree of infection. Lecithin bodies are the normal components of prostatic fluid in young men; their levels can indicate the state of prostatic function. Decreases in lecithin body may reflect various degrees of sexual 
dysfunction in males. Studies have shown that lecithin body is an index of severity of prostatitis and sexual function [18].

In the present study, Qianliexian capsule decreased the WBC count and increased number of lecithin bodies in ED patients, suggesting that the capsule can improve prostatic function and reduce infection. The occurrence of disease is related to immunity.

The Th1 cytokine, IFN- $\gamma$, participates in immune regulation: it induces multiple antigen presentation and cellular expression of $\mathrm{MHC}-\mathrm{I} / \mathrm{II}$ molecule, and it enhances the secretion of IL-1, tumor necrosis factor (TNF), IL-6 and IL-8. Thus, it boosts inflammatory reactions. In addition, IFN$Y$ increases the Th1/Th2 ratio. Studies have demonstrated that IL-8 level is closely related to acute infection, immune regulation and occurrence of tumors $[19,20]$. In this study, IFN-y and IL-8 levels of the patients were significantly decreased, relative to their levels before treatment, but after treatment, lower values were seen in the study group, relative to the controls. This indicates that Qianliexin capsule can be used to control inflammation in prostatitis patients with ED.

\section{CONCLUSION}

The results obtained in this study reveal that Qianliexin capsule can improve quality of life of patients with prostatitis complicated with ED. In addition, the results indicate that the use of Qianliexin capsule improves NIH-CPSI and IIEF5 scores, increases lecithin body number in EPS, decreases WBC count of EPS, and reduces IFN$Y$ and IL-8 levels in these patients. The treatment is also associated with a high degree of safety.

\section{DECLARATIONS}

\section{Conflict of Interest}

No conflict of interest associated with this work.

\section{Contribution of Authors}

We declare that this work was done by the author(s) named in this article and all liabilities pertaining to claims relating to the content of this article will be borne by the authors. All authors read and approved the manuscript for publication. Chen Xiaosong conceived and designed the study, Chen Xiaosong, Wang Ping collected and analysed the data, and Chen Xiaosong wrote the manuscript.

\section{REFERENCES}

1. Polackwich AS, Shoskes DA. Chronic prostatitis/chronic pelvic pain syndrome: a review of evaluation and therapy. Prostate Cancer Prostatic Dis 2016; 19(2): 132138.

2. Schaeffer EM. Re: Contemporary Management of Chronic Prostatitis/Chronic Pelvic Pain Syndrome. J Urol 2017; 197(5): 1275.

3. Hu LX, Zhu CH, Tu XS, Chen Y, Feng LI. Curative effect of small doses of tadalafil in the treatment for the chronic prostatitis combined with erectile dysfunction. Chin J Human Sex 2016; 25(2): 15-17.

4. Hou Er W, Wang L, Li X, Wang H. Clinical effect of tadalafil for treatment of chronic prostatitis combined erectile dysfunction. Chin J Human Sex 2017; 12(4): 2224.

5. Gao YQ, Wang $S H$, Jiang $S B$. Combined application of fufangxuanju capsule for the treatment of erectile dysfunction in chronic prostatitis: a Meta-analysis. Zhejiang Med J 2016; 38(24): 2000-2002.

6. Liang $D L, Y u N S$, Xie LH. Clinical study on traditional Chinese medicine differentiation combined with tamsulosin in the treatment of type III prostatitis with sexual dysfunction. Chin J Human Sex 2017; 26(12): 76-79.

7. Chen JH, Lin SH, Laing CW, Wu DJ. Therapeutic effect of Vardenafil de-escalation therapy on chronic prostatitis with erectile dysfunction. China J Mod Med 2018; 12(7): 99-104.

8. Zhang CH, Li YF, Qin GZ, Shen JH; Xu B, Zhao F, Zhang FG, Yang YJ, Chen TB, Ding SL, Zou JD. Clinical research on Qianlietongqiao Capsule in treating benign prostatic hyperplasia with kidney deficiency and stasis syndrome: A clinical observation of 87 cases. China J Tradit Chin Med Pharm 2016; 56(10): 4299-4302.

9. World Health Organization. Declaration of Helsinki. Br Med J 1996; 313(7070): 1448-1449.

10. Meier-Schroers M, Kukuk G, Wolter K, Decker G, Fischer $S$, Marx C, Traeber F, Sprinkart AM, Block W, Schild $\mathrm{HH}$, et al. Differentiation of prostatitis and prostate cancer using the Prostate Imaging-Reporting and Data System (PI-RADS). Eur J Radiol 2016; 85(7): 13041311.

11. Zhang $Y$, Lin $Q$, Cao $L P$. Treatment of chronic prostatitis/chronic pelvic pain syndrome by noninvasive prostatectomy combined with western medicine. Chin J Biochem Pharm 2017; 37(6): 109-110.

12. Li HJ, Kang DY. Prevalence of sexual dysfunction in men with chronic prostatitis/chronic pelvic pain syndrome: a meta-analysis. World J Urol 2016; 34(7): 1009-1017.

13. Ke $\mathrm{MH}$, Zhang $\mathrm{PL}$, Wang $X$, Wang $\mathrm{CH}$. Observation on the influences of Qianliexin capsule on chronic nonbacterial prostatitis. China J Tradit Chin Med Pharm 2016; 22(6): 2161-2163.

14. He XF, Yu C, Cao HW, Chen L, Xia WP, Feng YG. Clinical verifications of Honglu mixture on chronic 
prostatitis. Chin Tradit Patent Med 2016; 38(6):14341436.

15. Zhang CY, Yan L, Hong LI. Clinical efficacy of Xihuang Capsule on alleviating adverse reactions of chemotherapy in lung cancer. Guangxi Med J 2016; 38(5): 664-666.

16. Wang $D$, Gong $Z$, Zhang L, Shi JF, Hu CP, Cao P. Effects of Guizhifuling Capsule on Primary Dysmenorrhea Patients about Inflammation Factor. Chin $J$ Clin Pharm \& Thera 2016; 21(4): 441-444.

17. Liu W. Effects of acupuncture combined with Qiangjingzhuyu capsule on sperm motility in patient with asthenospermia. Hebei J Tradit Chin Med 2016; 38(8): 1213-1215.
18. Pan XF, LI B, Wang YF. A Study on Correlation of Leukocyte and Lecithin Body with Chronic Prostatitis by EPSRT. J Guiyang Med College 2015; 13(4): 404-406.

19. Birhanu BT, Lee JS, Lee SJ, Choi SH, Hossain MA, Park JY, Kim JC, Suh JW, Park SC. Immunomodulation of Lactobacillus pentosus, PL11 against Edwardsiella tarda, infection in the head kidney cells of the Japanese eel (Anguilla japonica). Fish Shellfish Immunol 2016; 54 : 466-472.

20. Zhang JC, Zheng XF, Chang ZH, Wang Y. Advancement of Regulatory $T$ Cell in the Immunomodulation of Human Immunodeficiency Virus Infection. Lett Biotechnol 2016; 27(2): 279-282. 